ESAIM: PROCEEDINGS AND SURVEYS, October 2015, Vol. 51, p. 193-211

A. Garivier et al, Editors

\title{
LONG TIME BEHAVIOR OF MARKOV PROCESSES AND BEYOND*
}

\author{
Florian Bouguet $^{1}$, Florent Malrieu $^{2}$, Fabien Panloup $^{3}$, Christophe PoqueT $^{4}$ and \\ JULIEN REYGNER ${ }^{5}$
}

\begin{abstract}
This note provides several recent progresses in the study of long time behavior of Markov processes. The examples presented below are related to other scientific fields as PDE's, physics or biology. The involved mathematical tools as propagation of chaos, coupling, functional inequalities, provide a good picture of the classical methods that furnish quantitative rates of convergence to equilibrium.

Résumé. Cet article présente plusieurs progrès récents dans l'étude du comportement en temps long de certains processus de Markov. Les exemples présentés ci-dessous sont motivés par différentes applications issues de la physique ou de la biologie. Les outils mathématiques employés, propagation du chaos, couplage, inégalités fonctionnelles, couvrent un large spectre des techniques disponibles pour obtenir des comportements en temps long quantitatifs.
\end{abstract}

\section{INTRODUCTION}

This note gathers several progresses in the study of the long time behavior of Markovian (and non Markovian) processes. The first section is dedicated to the study of stochastic differential equation driven by a fraction Brownian motion with a non constant diffusion matrix. This process is not Markovian but one can successfully adapt the coupling strategy to get quantitative long time estimates. In the second section, a piecewise deterministic Markov process arising linked to a stochastic algorithm is studied thanks to clever couplings of the paths. The last two sections stress the fruitful links between mean field interacting particle systems and non linear parabolic partial differential equations.

\section{RATE OF CONVERGENCE TO EQUILIBRIUM FOR FRACTIONAL SDES}

This part, which is a short version of [11], is devoted to the problem of the estimation of the rate of convergence to equilibrium of stochastic differential equations (SDEs) driven by a fractional Brownian motion ( $\mathrm{fBm}$ ). In this highly not Markovian setting, this problem has been first investigated by Hairer [17] who proved that, in the additive setting, the rate of convergence in total variation can be upper-bounded by $C t^{-\rho_{H}}$ where $\rho_{H}$ is a positive number depending on the Hurst parameter $H$ of the $\mathrm{fBm}$. But to our knowledge, there is no result in the multiplicative setting. Hence, we focus on the generalization of the existing additive results to the multiplicative case and obtain an extension of [17] when

* The authors thanks the Agence Nationale de la Recherche PIECE 12-JS01-0006-01 for its financial support.

${ }^{1}$ Institut de Recherche Mathématique de Rennes (UMR 6625 CNRS), Université de Rennes 1, Campus de Beaulieu, F-35042 Rennes Cedex, France.

${ }^{2}$ Laboratoire de Mathématiques et Physique Théorique (UMR CNRS 7350), Fédération Denis Poisson (FR CNRS 2964), Université FrançoisRabelais, Parc de Grandmont, 37200 Tours, France.

${ }^{3}$ Institut de Mathématiques de Toulouse (UMR CNRS 5219), Université Paul Sabatier \& INSA Toulouse, 135, av. de Rangueil, F-31077 Toulouse Cedex 4, France.

${ }^{4}$ Dipartimento di Matematica, Università degli studi di Roma Tor Vergata, Via della Ricerca Scientifica I-00133 Roma, Italy.

${ }^{5}$ Laboratoire de Physique (UMR CNRS 5672), École Normale Supérieure de Lyon, 46 allée d'Italie, Lyon, 69364, France.

(c) EDP Sciences, SMAI 2015 
$H>1 / 2$. The main novelty of this work is a development of some Foster-Lyapunov techniques in this non-Markovian setting, which allows us to put in place an asymptotic coupling scheme as that of [17] without resorting to deterministic contracting properties.

\subsection{Introduction}

We deal with an $\mathbb{R}^{d}$-valued process $\left(X_{t}\right)_{t \geq 0}$ which is a solution to the following SDE

$$
d X_{t}=b\left(X_{t}\right) d t+\sigma\left(X_{t}\right) d B_{t}
$$

where $b: \mathbb{R}^{d} \rightarrow \mathbb{R}^{d}$ and $\sigma: \mathbb{R}^{d} \rightarrow \mathbb{M}_{d, d}$ are (at least) continuous functions, and where $M_{d, d}$ is the set of $d \times d$ real matrices. In (1), $\left(B_{t}\right)_{t \geq 0}$ is a $d$-dimensional $\mathrm{fBm}$ with Hurst parameter $H \in\left(\frac{1}{2}, 1\right)$. Note that under some Hölder regularity assumptions on the coefficients (see e.g. [31] for background), (strong) existence and uniqueness hold for the solution to (1) starting from $x_{0} \in \mathbb{R}^{d}$.

Introducing the Mandelbrot-Van Ness representation of the fractional Brownian motion,

$$
B_{t}=\alpha_{H} \int_{-\infty}^{0}(-r)^{H-\frac{1}{2}}\left(d W_{r+t}-d W_{r}\right), \quad t \geq 0
$$

where $\left(W_{t}\right)_{t \in \mathbb{R}}$ is a two-sided $\mathbb{R}^{d}$-valued Brownian motion and $\alpha_{H}$ is a normalization coefficient depending on $H$, $\left(X_{t},\left(B_{s+t}\right)_{s \leq 0}\right)_{t \geq 0}$ can be realized through a Feller transformation $\left(\mathcal{Q}_{t}\right)_{t \geq 0}$ on the product space $\mathbb{R}^{d} \times \mathcal{W}$ where $\mathcal{W}$ is a suitable Hölder-type space on $(-\infty, 0]$ (we refer to [18] for more rigorous background on this topic). In particular, an initial distribution of this dynamical system is a distribution $\mu_{0}$ on $\mathbb{R}^{d} \times \mathcal{W}$. With probabilistic words, an initial distribution is the distribution of a couple $\left(X_{0},\left(B_{s}\right)_{s \leq 0}\right)$ where $\left(B_{s}\right)_{s \leq 0}$ is an $\mathbb{R}^{d}$-valued fBm on $(-\infty, 0]$.

Then, such an initial distribution is classically called an invariant distribution if it is invariant by the transformation $\mathcal{Q}_{t}$ for every $t \geq 0$. However, the concept of uniqueness of invariant distribution is slightly different from the classical setting. Actually, if $\bar{Q} \mu$ stands for the distribution of the whole process $\left(X_{t}^{\mu}\right)_{t \geq 0}$ with initial distribution $\mu$, one says that uniqueness of the invariant distribution holds if the stationary regime is unique (in other words, this concept of uniqueness corresponds to the classical one conditioned by the equivalence relation: $\mu \sim \nu \Longleftrightarrow \bar{Q} \mu \sim \bar{Q} \nu$, see [18] for background). We refer to [17-19] for criteria of uniqueness in different settings: additive noise, multiplicative noise with $H>1 / 2$ and multiplicative noise with $H \in(1 / 3,1 / 2)$ in the last one (in an hypoelliptic context).

The additive result of [17] is obtained by a coupling strategy that we briefly recall here. Classically, coupling two paths issued of $\mu_{0}$ and $\mu$ where the second one denotes an invariant distribution of $\left(\mathcal{Q}_{t}\right)_{t>0}$ consists in finding a stopping time $\tau_{\infty}$ such that $\left(X_{t+\tau_{\infty}}^{\mu_{0}}\right)_{t \geq 0}=\left(X_{t+\tau_{\infty}}^{\mu}\right)_{t \geq 0}$ (so that the rate of convergence in total variation can be derived from some bounds on $\left.\mathbb{P}\left(\tau_{\infty}>t\right), t \geq 0\right)$. Now, let us detail the strategy. First, one classically waits that the paths get close. Then, at each trial, the coupling attempt is divided in two steps. First, one tries in Step 1 to stick the positions on an interval of length 1. Then, in Step 2, one tries to ensure that the paths stay stuck until $+\infty$. Actually, oppositely to the Markovian case where the paths stay naturally together after a clustering (by putting the same noise on each coordinate), the main difficulty here is that, due to the memory, staying together is costly. In other words, this property can be ensured only with the help of a non trivial coupling of the noises. One thus talks of asymptotic coupling. If one of the two previous steps fails, we will begin a new attempt but only after a (long) waiting time which is called Step 3. During this step, one again waits that the paths get close but one also expects the memory of the coupling cost to vanish sufficiently in order to begin the new trial with a weak weight of the memory.

In the previous construction, the fact that $\sigma$ is constant is fundamental for ensuring the two following properties:

- If two paths $B^{1}$ and $B^{2}$ of the fBm differ from a drift term, then two paths $X^{1}$ and $X^{2}$ of (1) respectively directed by $B^{1}$ and $B^{2}$ also differ from a drift term, which allows in particular to use Girsanov Theorem to build the coupling in Step 1.

- Under some "convexity" assumptions on the drift apart from a compact set, two paths $X^{1}$ and $X^{2}$ directed by the same fBm (or more precisely, by two slightly different paths) get closer and the distance between the two paths can be controlled deterministically. 
In the current work, $\sigma$ is not constant and the two above properties are no longer valid. The challenge then is to extend the applicability of the previous coupling scheme to such a situation. The replacement of each of the above properties requires us to deal with different (though related) difficulties. In order to be able to extend the Girsanov argument used in Step 1 to a non constant $\sigma$, we will restrain ourselves to diffusion coefficients for which some injective function of two copies of the process differs by a drift term whenever their driving fBm do. A natural assumption on $\sigma$ granting the latter property is that $x \mapsto \sigma^{-1}(x)$ is (well-defined and is) a Jacobian matrix. This will be the setting of the present paper.

As concerns a suitable substitution of the second lacking property, a natural (but to our knowledge so far not explored) idea is to try to extend Meyn-Tweedie techniques (see e.g. [10] for background) to the fractional setting. More precisely, even if the paths do not get closer to each other deterministically, one could expect that some Lyapunov assumption could eventually make the two paths return in some compact set simultaneously. The main contribution of the present paper is to incorporate such a Lyapunov-type approach into the study of long-time convergence in the fractional diffusion setting. As one could expect, compared to the Markovian case, the problem is much more involved. Actually, the return time to a compact set after a (failed) coupling attempt does not only depend on the positions of the processes after it, but also on all the past of the fBm. Therefore, in order that the coupling attempt succeeds with lower-bounded probability, one needs to establish some controls on the past behavior of the fBms that drive the two copies of the process, conditionally to the failure of the previous attempts. This point is one of the main difficulties of the paper, since, in the corresponding estimates, we carefully have to take into account all the deformations of the distribution that previously failed attempts induce. Then, we show that after a sufficiently long waiting time, conditionally on previous fails the probability that the two paths be in a compact set and that the influence of past noise on the future be controlled, is lower-bounded. Bringing all the estimates together yields a global control of the coupling time and a rate of convergence which is similar to the one in [17] in the additive noise case.

\subsection{Assumptions and Main Result}

Remind that in the whole section it is assumed that $H \in(1 / 2,1)$. We begin by a condition for the existence and uniqueness of solutions for (1):

$\left(\mathbf{H}_{\mathbf{0}}\right): b$ is a locally Lipschitz and sublinear function and $\sigma$ is a bounded $(1+\gamma)$-Lipschitz continuous function with $\gamma \in\left(\frac{1}{H}-1,1\right]$ (i.e. $\sigma$ is a $\mathcal{C}^{1}$-function whose partial derivatives are bounded and globally $\gamma$-Hölder-continuous).

Before introducing the second assumption, let us give a definition. We say that a function $V: \mathbb{R}^{d} \rightarrow \mathbb{R}$ is essentially quadratic if it is a positive $\mathcal{C}^{1}$-function such that $\nabla V$ is Lipschitz continuous and such that

$$
\liminf _{|x| \rightarrow \infty} \frac{V(x)}{|x|^{2}}>0 \quad \text { and } \quad|\nabla V| \leq C \sqrt{V} \quad(C>0) .
$$

In order to ensure the existence of the invariant distribution, we now introduce a Lyapunov-stability assumption $\left(\mathbf{H}_{\mathbf{1}}\right)$ through such a function $V$ :

$\left(\mathbf{H}_{\mathbf{1}}\right)$ : There exists an essentially quadratic function $V: \mathbb{R}^{d} \rightarrow \mathbb{R}$, there exist some positive $\beta_{0}$ and $\kappa_{0}$ such that

$$
\forall x \in \mathbb{R}^{d}, \quad(\nabla V(x) \mid b(x)) \leq \beta_{0}-\kappa_{0} V(x) .
$$

Remark 1.1 (Comparison to the Markovian case). For the coupling strategy, the above assumption will be certainly used to ensure that the paths live in a compact set with a high probability. Note that in the classical diffusion setting, such a property holds with some less restrictive Lyapunov assumptions. Here, the assumptions $\left(\mathbf{H}_{\mathbf{0}}\right)$ and $\left(\mathbf{H}_{\mathbf{1}}\right)$ essentially allow us to consider only (attractive) drift terms whose growth is linear at infinity. This more restrictive condition can be understood as a consequence of the lack of martingale property for the integrals driven by fBms, which leads in fact to a more important contribution of the noise component.

Remark 1.2 (Comparison to previous results). In [17], the corresponding assumption is a contraction condition out of a compact set: for any $x, y,(b(x)-b(y) \mid x-y) \leq \beta_{0}-\kappa_{0}|x-y|^{2}$. This means that even in the constant case, our work can cover some new cases. For instance, if $d=2$ and $b(z)=-z-\rho \cos \left(\theta_{z}\right) z^{\perp}$ (where $\rho \in \mathbb{R}, \theta_{z}$ is the angle of $z$ and $z^{\perp}$ is its normal vector), Assumption $\left(\mathbf{H}_{\mathbf{1}}\right)$ holds whereas one can check that the contraction condition is not satisfied if $\rho>2$. 
When the paths are in this compact set, one tries classically to couple them with positive probability. But, as mentioned before, the specificity of the non-Markovian setting is that the coupling attempt generates a cost for the future (in a sense made precise later). In order to control this cost or more precisely in order to couple the paths with the help of a controlled drift term, we need to ensure the next assumption:

$\left(\mathbf{H}_{\mathbf{2}}\right) \forall x \in \mathbb{R}^{d}, \sigma(x)$ is invertible and there exists a $\mathcal{C}^{1}$-function $h=\left(h_{1}, \ldots, h_{d}\right): \mathbb{R}^{d} \rightarrow \mathbb{R}^{d}$ such that the Jacobian matrix $\nabla h=\left(\partial_{x_{j}} h_{i}\right)_{i, j \in\{1, \ldots, d\}}$ satisfies $\nabla h(x)=\sigma^{-1}(x)$ and such that $\nabla h$ is a locally Lipschitz function on $\mathbb{R}^{d}$.

Remark 1.3 (On the regularity of the diffusion matrix). Under $\left(\mathbf{H}_{\mathbf{0}}\right)$ and $\left(\mathbf{H}_{\mathbf{2}}\right), h$ is a global $\mathcal{C}^{1}$-diffeomorphism from $\mathbb{R}^{d}$ to $\mathbb{R}^{d}$. Indeed, under these assumptions, $\nabla h$ is invertible everywhere and $x \mapsto[(\nabla h)(x)]^{-1}=\sigma(x)$ is bounded on $\mathbb{R}^{d}$. Then, the property (which will be important in the sequel), follows from the Hadamard-Lévy theorem (see e.g. [35]).

As mentioned before, the main restriction here is to assume that $x \mapsto \sigma^{-1}(x)$ is a Jacobian matrix. However, note that there is no assumption on $h$ (excepted smoothness). In particular, $\sigma^{-1}$ does not need to bounded. This allows us to consider for instance some cases where $\sigma$ vanishes at infinity.

Let us exhibit some simple classes of SDEs for which $\left(\mathbf{H}_{\mathbf{2}}\right)$ is fulfilled. First, it contains the class of non-degenerated SDEs for which each coordinate is directed by one real-valued fBm. More precisely, if for every $i \in\{1, \ldots, d\}$,

$$
d X_{t}^{i}=b_{i}\left(X_{t}^{1}, \ldots, X_{t}^{d}\right) d t+\sigma_{i}\left(X_{t}^{1}, \ldots, X_{t}^{d}\right) d B_{t}^{i}
$$

where $\sigma_{i}: \mathbb{R}^{d} \rightarrow \mathbb{R}$ is a $\mathcal{C}^{1}$ positive function, Assumption $\left(\mathbf{H}_{\mathbf{2}}\right)$ holds. Now, let us also remark that since, for a given constant matrix, $\nabla(P h)=P \nabla h$, we have the following equivalence:

$$
\exists h \text { such that } \nabla h=\sigma^{-1} \Longleftrightarrow \exists \tilde{h}, \exists \text { an invertible matrix } P \text { such that } \sigma^{-1}=P \nabla \tilde{h} \text {. }
$$

One deduces from this property that $\left(\mathbf{H}_{2}\right)$ also holds true if:

$$
\sigma(x)=P \operatorname{Diag}\left(\sigma_{1}\left(x_{1}, \ldots, x_{d}\right), \ldots, \sigma_{d}\left(x_{1}, \ldots, x_{d}\right)\right)
$$

where $P$ is a given invertible $d \times d$-matrix and for every $i \in\{1, \ldots, d\} \sigma_{i}$ has the same properties as before.

We are now able to state our main result. One denotes by $\mathcal{L}\left(\left(X_{t}^{\mu_{0}}\right)_{t \geq 0}\right)$ the distribution of the process on the set $\mathcal{C}\left([0,+\infty), \mathbb{R}^{d}\right)$ starting from an initial distribution $\mu_{0}$ and by $\overline{\mathcal{Q}} \mu$ the distribution of the stationary solution (starting from an invariant distribution $\mu$ ). The distribution $\bar{\mu}_{0}(d x)$ denotes the first marginal of $\mu_{0}(d x, d w)$.

Theorem 1.4. Let $H \in(1 / 2,1)$. Assume $\left(\mathbf{H}_{\mathbf{0}}\right),\left(\mathbf{H}_{\mathbf{1}}\right)$ and $\left(\mathbf{H}_{\mathbf{2}}\right)$. Then, existence and uniqueness hold for the invariant distribution $\mu$ (up to equivalence). Furthermore, for every initial distribution $\mu_{0}$ for which there exists $r>0$ such that $\int|x|^{r} \bar{\mu}_{0}(d x)<\infty$, for each $\varepsilon>0$ there exists $C_{\varepsilon}>0$ such that

$$
\left\|\mathcal{L}\left(\left(X_{t+s}^{\mu_{0}}\right)_{s \geq 0}\right)-\overline{\mathcal{Q}} \mu\right\|_{\mathrm{TV}} \leq C_{\varepsilon} t^{-\left(\frac{1}{8}-\varepsilon\right)}
$$

Remark 1.5. In the previous result, the main contribution is the fact that one is able to recover the rates of the additive case. Existence and uniqueness results are not really new. However, compared with the assumptions of [18], one observes that when $x \mapsto \sigma^{-1}(x)$ is a Jacobian matrix (assumption which does not appear in [18]), our other assumptions are slightly less constraining. In particular, $b$ is assumed to be locally Lipschitz and sublinear (instead of Lipschitz continuous) and, as mentioned before, $x \mapsto \sigma^{-1}(x)$ does not need to bounded.

Some ingredients of the proof. The aim of this section is to give some ideas of the proof. As explained above, the scheme is similar to that of [17]. The starting point is to consider a couple $(X, \tilde{X})$ of solutions to (1) with respective driving fBms denoted by $B$ and $\tilde{B}$. The underlying innovation processes are denoted by $W$ and $\tilde{W}$. For the sake of simplicity, assume that $X_{0}=x \in \mathbb{R}^{d}$ and that the initial condition of $\tilde{X}$ is the invariant distribution $\mu$. For every $k \geq 1$, denote by $\tau_{k-1}$, the starting time of the $k^{t h}$-coupling attempt and by $\Delta \tau_{k}$ its duration. If the coupling is successful, $X$ and $\tilde{X}$ get stuck after Step 1, i.e. $\tau_{k-1}+1$. We thus define $\tau_{\infty}:=\tau_{k^{*}-1}+1$ where $k^{*}:=\inf \left\{k \geq 1, \Delta \tau_{k}=+\infty\right\}$. By construction,

$$
\forall t \geq 0, \quad\left\|\mathcal{L}\left(\left(X_{t+s}^{\mu_{0}}\right)_{s \geq 0}\right)-\overline{\mathcal{Q}} \mu\right\|_{\mathrm{TV}} \leq \mathbb{P}\left(\tau_{\infty}>t\right)
$$


Furthermore, using that

$$
\forall t \geq 1, \quad \mathbb{P}\left(\tau_{\infty}>t-1\right)=\mathbb{P}\left(\tau_{0}+\sum_{k=1}^{+\infty} \Delta \tau_{k} 1_{k^{*}>k}>t\right)
$$

it can be shown (see [11], section 5) that the problem can be more or less reduced to the control (uniform in $k$ ) of :

$$
\mathbb{P}\left(\Delta \tau_{k}<+\infty \mid \Delta \tau_{k-1}<+\infty, \mathcal{F}_{\tau_{k-1}}\right) \quad \text { and } \quad \mathbb{E}\left[\left|\Delta \tau_{k}\right|^{p} \mid \Delta \tau_{k}, \mathcal{F}_{\tau_{k-1}}\right]
$$

where $\left(\mathcal{F}_{t}\right)_{t \geq 0}$ is the usual augmentation of $\left(\left(\sigma\left(W_{s}, \tilde{W}_{s}\right)\right)_{s \leq t}\right)_{t \geq 0}$ and $p \in(0,1)$ is a real that one will try to maximize (as suggested by Theorem 1.4, this expectation will be finite as soon as $p<1 / 8$ ).

$(K, \alpha)$-admissibility. The quantities defined previously can be controlled only if the positions of each component and the past of their noise satisfy some conditions at the beginning of the attempt. One will talk about $(K, \alpha)$-admissibility. In order to define this concept, we now assume that $W$ and $\tilde{W}$ differ from a drift term denoted by $g_{w}: d W_{t}=d \tilde{W}_{t}+g_{w}(t) d t$. The function $g_{w}$ will be supposed to be null before $\tau_{0}$, i.e. before the first attempt and also during Step 3 . In order to quantify the impact of $g_{w}$ on the future attempts, one introduces an operator $\mathcal{R}_{T}$ defined (when it makes sense) by: for all $T \geq 0$ and for all $g: \mathbb{R} \rightarrow \mathbb{R}$

$$
\left(\mathcal{R}_{T} g\right)(t)=\int_{-\infty}^{0} \frac{t^{\frac{1}{2}-H}(T-s)^{H-\frac{1}{2}}}{t+T-s} g(s) d s, \quad t \in(0,+\infty) .
$$

The admissibility condition can be then defined as follows:

Definition 1.6. Let $K$ and $\alpha$ be some positive numbers and $\tau$ be a $\left(\mathcal{F}_{t}\right)_{t \geq 0}$-stopping time. One says that the system is $(K, \alpha)$-admissible at time $\tau$ if $\tau(\omega)<+\infty$ and if $\left(X_{\tau}^{1}(\omega), X_{\tau}^{2}(\omega),\left(W^{1}(\omega), W^{2}(\omega)\right)_{t \leq \tau}\right)$ satisfies :

$$
\sup _{T \geq 0} \int_{0}^{+\infty}(1+t)^{2 \alpha}\left|\left(\mathcal{R}_{T} g_{w}^{\tau}\right)(t)\right|^{2} d t \leq 1 \quad \text { where } g_{w}^{\tau}(.)=g_{w}(.+\tau)
$$

is the shift of drift term between $W$ and $\tilde{W}$, and if $\left(X_{\tau}^{1}(\omega), X_{\tau}^{2}(\omega),\left(W^{1}(\omega), W^{2}(\omega)\right)_{t \leq \tau}\right) \in \Omega_{K, \alpha, \tau}$ where

$$
\Omega_{K, \alpha, \tau}:=\left\{\left|X_{\tau}^{1}(\omega)\right| \leq K,\left|X_{\tau}^{2}(\omega)\right| \leq K, \varphi_{\tau, \varepsilon_{\theta}}\left(W^{1}(\omega)\right) \leq K \quad \text { and } \quad \varphi_{\tau, \varepsilon_{\theta}}\left(W^{2}(\omega)\right) \leq K\right\},
$$

and $\varepsilon_{\theta}=\frac{H-\theta}{2}$ with $\theta \in(1 / 2, H)$ and for all $\varepsilon>0$,

$$
\varphi_{\tau, \varepsilon}(w)=\sup _{\tau \leq s \leq t \leq \tau+1}\left|\frac{1}{t-s} \int_{-\infty}^{\tau-1}(t-r)^{H-\frac{1}{2}}-(s-r)^{H-\frac{1}{2}} d w_{r}\right|+\sup _{\tau-1 \leq u<v \leq \tau} \frac{|w(v)-w(u)|}{|v-u|^{\frac{1}{2}-\varepsilon}} .
$$

The $(K, \alpha)$-admissibility is a sufficient condition to ensure that the coupling succeeds with lower-bounded probability (i.e. such that the paths remain stuck until infinity). More precisely, under this condition, one is able to show that there exists $\delta_{0}>0$ such that for every $k \geq 1$,

$$
\mathbb{P}\left(\Delta \tau_{k}=+\infty \mid\left\{\Delta_{\tau_{k-1}}<+\infty\right\} \cap \Omega_{K, \alpha, \tau_{k-1}}\right) \geq \delta_{0}
$$

Condition (3), which comes from [17], is adapted for Step 2, ensuring that if $X_{\tau_{k-1}+1}=\tilde{X}_{\tau_{k-1}+1}$ (i.e. if Step 1 succeeds), one can build some couples $(W, \tilde{W})$ on successive intervals of lengths $2^{\alpha N}$ such that the probability that the paths remain stuck until infinity is lower-bounded. The second condition plays an important role for Step 1 . When $\omega \in \Omega_{K, \alpha, \tau_{k-1}}$, the cost to stick the paths can be uniformly controlled under Assumption $\left(\mathbf{H}_{\mathbf{2}}\right)$. Then, one of the main difficulties of the proof in the multiplicative case is to show that the probability of $(K, \alpha)$-admissibility is also lower-bounded: one needs to show that there exists $\delta_{1}>0$ such that for every $k \geq 1$,

$$
\mathbb{P}\left(\Omega_{K, \alpha, \tau_{k}} \mid \Delta \tau_{k-1}<+\infty\right) \geq \delta_{1}
$$


The proof of this property is achieved in two main steps. In the first one, one needs to control the increments of $W$ and $\tilde{W}$ before $\tau_{k}$, conditionally to the failure of the previous attempts. This control requires a sharp knowledge of the construction of the innovations used in Steps 1 and 2 in order to identify the distortions generated by each scenario of failed attempt. Then, plugging these controls into the Mandelbrot-Van Ness representation allows us to estimate the impact of these distortions on the future of the increments of the fBm (see Lemmas 4.5, 4.6 et 4.7 of [11] for more details).

With the help of the Lyapunov assumption $\left(\mathbf{H}_{\mathbf{1}}\right)$, one tries in the second step to upper-bound the quantity $\mathbb{E}\left[V^{r}\left(X_{\tau_{k}}\right)+\right.$ $V^{r}\left(\tilde{X}_{\tau_{k}}\right) \mid \Delta \tau_{k}<+\infty$ ] (for a positive power $r$ ). Conditionally to the first step, the keypoint is the following contraction property (Proposition 4.4 of [11]) : if $\left(\mathbf{H}_{\mathbf{0}}\right)$ and $\left(\mathbf{H}_{\mathbf{1}}\right)$ hold, then, for every $\theta \in(1 / 2, H)$, there exists $\bar{\rho} \in(0,1), C>0$ and $r>0$ such that for every starting point $x \in \mathbb{R}^{d}$,

$$
V^{r}\left(X_{1}\right) \leq \bar{\rho} V^{r}(x)+C\left(1+\left\|B^{H}\right\|_{\theta}^{0,1}\right) \quad \text { where } \quad\|B\|_{\theta}^{0,1}=\sup _{0<s<t<1} \frac{\left|B_{t}^{H}-B_{s}^{H}\right|}{(t-s)^{\theta}} .
$$

We refer to [11] for the complete proof.

\section{THE PENALIZED BANDIT PROCESS}

The two-armed bandit algorithm is a theoretical procedure to choose asymptotically the most profitable arm of a slot machine, or bandit; it was also used in the fields of mathematical psychology and of engineering. This algorithm has been widely studied, for instance in [27,29]. The key idea is to use a (deterministic) sequence of learning rates, rewarding an arm if it delivers a gain. Depending on the speed of convergence to 0 of this sequence, the algorithm is often faillible (it would not always select asymptotically the right arm, see [29]).

It is possible to improve its results and ensure infaillibility by introducing penalties when the arm does not deliver a gain: this modification is called the penalized bandit algorithm, and it is studied in [28]. The authors show that, with a correct choice of penalties and rewards, and with the appropriate renormalization, the algorithm converges weakly to a probability measure $\pi$, which is the stationary distribution of the Piecewise Deterministic Markov Process with following infinitesimal generator

$$
\mathcal{L} f(x)=(1-p-p x) f^{\prime}(x)+q x \frac{f(x+g)-f(x)}{g} .
$$

where $0<q<p<1$ being the respective probabilities of gain of the two arms and the positive parameter $g$ runs the asymptotic behaviour of the sequences of the rewards and penalties (see Section 3 in [28] for more details). This process is also studied in [13]. For the sake of simplicity, we set $g=1$ in the sequel. Moreover, since the interval $[0,(1-p) / p)$ is transient, computations are easier if we study the translated process $Y=X-\frac{1-p}{p}$, driven by the following generator:

$$
\mathcal{L}^{Y} f(y)=-p y f^{\prime}(y)+q\left(y+\frac{1-p}{p}\right)(f(y+1)-f(y)) .
$$

It is possible to deduce the dynamics of the process from the generator (see [9]): between the jumps, $Y$ evolves as the solution of the ODE $y_{t}^{\prime}=-p y_{t}$, and it jumps with jump rate $t \mapsto \zeta\left(Y_{t}\right)=q\left(Y_{t}+\frac{1-p}{p}\right)$ from $Y_{t}$ to $Y_{t}+1$.

In [28], the authors show that $\pi$ admits a density with support $[(1-p) / p,+\infty)$ and exponential moments of order up to $u_{M}$, where $u_{M}$ is the unique positive solution of the equation

$$
\frac{\exp \left(u_{M}\right)-1}{u_{M}}=\frac{p}{q}
$$

We shall prove the latter too, but with a different argument (see Remark 2.2).

In the sequel, we denote by penalized bandit process the process with initial distribution $\mu_{0}$ following the dynamics of $\mathcal{L}^{Y}$, and by $\mu_{t}$ its law at time $t \geq 0$. This section is devoted to the long time behavior of this process with respect to Wasserstein and total variation distances. The estimates rely on the construction of explicit couplings. This approach is closely related to the paper [7] which is dedicated to the study of a Piecewise Deterministic Markov Process related to a pharmacokinetic model introduced in [2]. 


\subsection{Wasserstein convergence}

Firstly, let us recall the definitions of Wasserstein and total variation distances between two measures:

$$
\begin{aligned}
\mathrm{W}_{n}(\mu, \nu) & =\inf \left\{\mathbb{E}\left[|X-Y|^{n}\right]^{\frac{1}{n}}:(X, Y) \text { coupling of } \mu \text { and } \nu\right\}, \\
\|\mu-\nu\|_{\mathrm{TV}} & =\inf \{\mathbb{P}(X \neq Y):(X, Y) \text { coupling of } \mu \text { and } \nu\} .
\end{aligned}
$$

In the following, let $\mu_{0}$ and $\widetilde{\mu}_{0}$ be two probabilities on $\mathbb{R}_{+}$. The following proposition holds:

Proposition 2.1. We have, for all $t \geq 0$

$$
\mathrm{W}_{1}\left(\mu_{t}, \widetilde{\mu}_{t}\right) \leq \mathrm{W}_{1}\left(\mu_{0}, \widetilde{\mu}_{0}\right) e^{-(p-q) t} .
$$

Proof. Let $(Y, \widetilde{Y})$ be generated by

$$
\begin{aligned}
\mathcal{L}_{2}^{Y} f(y, \widetilde{y})= & -p y \partial_{y} f(y, \widetilde{y})-p \widetilde{y} \partial_{y} f(y, \widetilde{y}) \\
& +q(y-\widetilde{y})(f(y+1, \widetilde{y})-f(y, \widetilde{y}))+q\left(\widetilde{y}+\frac{1-p}{p}\right)(f(y+1, \widetilde{y}+1)-f(y, \widetilde{y})),
\end{aligned}
$$

for $y \geq \widetilde{y}$, and of symetric expression for $\widetilde{y} \geq y$, and such that $\left(Y_{0}, \widetilde{Y}_{0}\right)$ is a coupling of $\left(\mu_{0}, \widetilde{\mu}_{0}\right)$ realizing $\mathrm{W}_{1}\left(\mu_{0}, \widetilde{\mu}_{0}\right)=$ $\mathbb{E}\left[\left|Y_{0}-\widetilde{Y}_{0}\right|\right]$. It is easy to check that (9) reduces to (6) if $f$ depends only on $y$ or $\widetilde{y}$, which means that $\left(Y_{t}, \widetilde{Y}_{t}\right)_{t \geq 0}$ generated with $\mathcal{L}_{2}^{Y}$ is a coupling of $\left(\mu_{t}, \widetilde{\mu}_{t}\right)_{t \geq 0}$. With this coupling, either the higher process jumps alone or the jump is simultaneous. It is easy to check that this coupling is monotonous, i.e. for all $t \geq 0,\left(Y_{t}-\widetilde{Y}_{t}\right)\left(Y_{0}-\widetilde{Y}_{0}\right) \geq 0$. Monotonicity comes from the fact that the higher process jumps more often but stays above the other since the jumps are positive. Assume that $Y_{0} \geq \widetilde{Y}_{0}$. By monotonicity, we have, for all $t \geq 0$,

$$
\mathbb{E}\left[\left|Y_{t}-\tilde{Y}_{t}\right|\right]=\mathbb{E}\left[Y_{t}\right]-\mathbb{E}\left[\tilde{Y}_{t}\right]
$$

so all we have to do is to study $h: t \mapsto \mathbb{E}\left[Y_{t}\right]$. With $f(y)=y$, (6) leads to $\mathcal{L} f(y)=\frac{q(1-p)}{p}-(p-q) y$, and, by Dynkin formula, the function $h$ satisfies the ordinary differential equation $h^{\prime}(t)=\frac{q(1-p)}{p}-(p-q) h(t)$. One deduces immediately that

$$
\mathbb{E}\left[Y_{t}\right]=\frac{q(1-p)}{p(p-q)}+\left(\mathbb{E}\left[Y_{0}\right]-\frac{q(1-p)}{p(p-q)}\right) \mathrm{e}^{-(p-q) t},
$$

(recall that $p>q$ ). Then,

which leads directly to (8)

$$
\mathbb{E}\left[\left|Y_{t}-\tilde{Y}_{t}\right|\right]=\mathbb{E}\left[Y_{0}-\tilde{Y}_{0}\right] \mathrm{e}^{-(p-q) t},
$$

Remark 2.2. The Dynkin formula is a powerful tool for studying the moments of Markov processes. One can use it with $f(y)=\mathrm{e}^{u y}$ to study the Laplace transform of the transient process $\psi(t, u)=\mathbb{E}\left[\mathrm{e}^{u Y_{t}}\right]$. We have

$$
\mathcal{L}^{Y} f(y)=q \frac{1-p}{p}\left(e^{u}-1\right) f(y)+\left(q\left(e^{u}-1\right)-u p\right) y f(y),
$$

so $\psi$ satisfies the following PDE:

$$
\partial_{t} \psi(t, u)=q \frac{1-p}{p}\left(e^{u}-1\right) \psi(t, u)+\left(q\left(e^{u}-1\right)-u p\right) \partial_{u} \psi(t, u) .
$$


If $\mu_{0}$ is the invariant measure $\pi$, then $\partial_{t} \psi(t, u)=0$, and the Laplace transform $u \mapsto \psi(u)$ is solution of the following ODE

$$
\partial_{u}(\log (\psi(u)))=\frac{q \frac{1-p}{p}\left(\mathrm{e}^{u}-1\right)}{u p-q\left(\mathrm{e}^{u}-1\right)},
$$

and the right-hand side is finite for $u \in\left[0, u_{M}\right)$, when $u_{M}$ is the solution of Equation (7).

Note that the set of polynomials of degree $n$ is stable under the action of $\mathcal{L}^{Y}$. This is an important property, since it theoretically enables us to compute the moments of $Y_{t}$ by induction, with the help of Dynkin formula, just as we did for the first moment in the proof of Proposition 2.1. Similarly, it is possible to study the function $h_{n}(t)=\mathbb{E}\left[\left|Y_{t}-\widetilde{Y}_{t}\right|^{n}\right]$ which provides an upper bound of $\mathrm{W}_{n}\left(\mu_{t}, \widetilde{\mu}_{t}\right)$. Indeed, we have, for $f(y, \widetilde{y})=|y-\widetilde{y}|^{n}$,

$$
\mathcal{L}_{2}^{Y} f(y, \widetilde{y})=-n(p-q)|y-\widetilde{y}|^{n}+q \sum_{k=0}^{n-2}\left(\begin{array}{l}
n \\
k
\end{array}\right)|y-\widetilde{y}|^{k+1},
$$

so

$$
h_{n}^{\prime}(t)=-n(p-q) h_{n}(t)+q \sum_{k=0}^{n-2}\left(\begin{array}{l}
n \\
k
\end{array}\right) h_{k+1}(t) .
$$

Then, using Gronwall lemma easily leads to check, by induction, that $h_{n}(t)=\mathcal{O}\left(\mathrm{e}^{-n(p-q) t}\right)$. Which leads to the following result:

Proposition 2.3. For all $n \in \mathbb{N}^{\star}$, there exists a positive constant $C$ such that, for all $t \geq 0$,

$$
\mathrm{W}_{n}\left(\mu_{t}, \widetilde{\mu}_{t}\right) \leq C e^{-(p-q) t} .
$$

\subsection{Total variation convergence}

In the case of the penalized bandit process, total variation convergence is slightly harder than in [7], since the jumps are always of amplitude range 1. Instead, we are going to use the arguments introduced in [1], based on the following observation: if $Y$ and $\widetilde{Y}$ are close enough, we can make them jump, not simultaneously like before, but with a slight delay for one of the copies, which would make it jump on the other one, as illustrated in Figure 1.

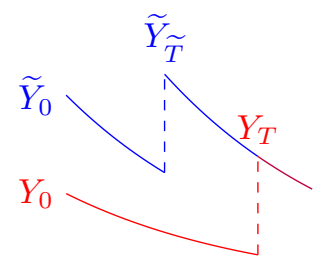

FIGURE 1. Expected behaviour of the coalescent coupling for the penalized bandit process.

In the following, denote by $\tau=\inf \left\{t \geq 0: \forall s \geq 0, Y_{t+s}=\widetilde{Y}_{t+s}\right\}$ the coalescence time of $Y$ and $\tilde{Y}$. The goal of the sequel is to obtain exponential moments for $\tau$ (which may happen for correct couplings) and then use the total variation classic coupling inequality:

We have the following lemma:

$$
\left\|\mu_{t}-\widetilde{\mu}_{t}\right\|_{\mathrm{TV}} \leq \mathbb{P}\left(Y_{t} \neq \tilde{Y}_{t}\right) \leq \mathbb{P}(\tau>t) .
$$

Lemma 2.4. Assume there exist positive constants $\bar{y}<+\infty, \bar{\varepsilon}<1$ such that $Y_{0}, \widetilde{Y}_{0} \leq \bar{y}$ and $\left|Y_{0}-\widetilde{Y}_{0}\right| \leq \bar{\varepsilon}$. Then, there exist a coupling $\left(Y_{t}, \widetilde{Y}_{t}\right)_{t \geq 0}$ of $\left(\mu_{t}, \widetilde{\mu}_{t}\right)_{t \geq 0}$ and an explicit positive constant $C(\bar{y}, \bar{\varepsilon})<+\infty$ such that, for all $t>0$,

$$
\mathbb{P}(\tau>t) \leq C(\bar{y}, \bar{\varepsilon})\left(\exp \left(-\frac{q(1-p)}{p} t\right)+\bar{\varepsilon}\right) .
$$


Proof. First, assume that $Y_{0}$ and $\widetilde{Y}_{0}$ are deterministic, and denote by $y=Y_{0}, \varepsilon=\widetilde{Y}_{0}-y$. We assume w.l.o.g. that $\varepsilon>0$. Let $T$ (resp. $\widetilde{T}$ ) be the first jump time of the process $Y$ (resp. $\widetilde{Y}$ ). Following the heuristics of Figure 1, it is straightforward that

Easy computations lead to

$$
Y_{T}=\widetilde{Y}_{T} \Leftrightarrow T=\frac{1}{p} \log \left(\varepsilon+\mathrm{e}^{p \widetilde{T}}\right) .
$$

$$
\mathbb{P}\left(\frac{1}{p} \log \left(\varepsilon+\mathrm{e}^{p \widetilde{T}}\right) \leq s\right)=\mathbb{P}\left(\widetilde{T} \leq \frac{1}{p} \log \left(\mathrm{e}^{p s}-\varepsilon\right)\right)=1-\Phi_{y}(s, \varepsilon),
$$

with

$$
\Phi_{y}(s, \varepsilon)=\exp \left(-\frac{q}{p}\left(\frac{1-p}{p} \log \left(\mathrm{e}^{p s}-\varepsilon\right)+(y+\varepsilon)\left(1-\frac{1}{\mathrm{e}^{p s}-\varepsilon}\right)\right)\right) .
$$

As a consequence, the random variables $T$ and $\frac{1}{p} \log (\varepsilon+\exp (p \widetilde{T}))$ admit densities w.r.t. the Lebesgue measure, which are respectively $f_{y}(\cdot, 0)$ and $f_{y}(\cdot, \varepsilon)$, with, for all $s \geq 0$,

$$
f_{y}(s, \varepsilon)=\frac{q \mathrm{e}^{p s}}{p}\left(\frac{1-p}{\mathrm{e}^{p s}-\varepsilon}+\frac{p(y+\varepsilon)}{\left(\mathrm{e}^{p s}-\varepsilon\right)^{2}}\right) \Phi_{y}(s, \varepsilon) .
$$

Let $T$ and $\frac{1}{p} \log (\varepsilon+\exp (p \widetilde{T}))$ follow the $\gamma$-coupling (the coupling minimizing the total variation of their laws, see [30]). It is not hard to deduce from the very construction of this coupling the following equality:

$$
\mathbb{P}\left(T=\frac{1}{p} \log \left(\varepsilon+\mathrm{e}^{p \widetilde{T}}\right), T<t\right)=\int_{0}^{t} f_{y}(s, 0) \wedge f_{y}(s, \varepsilon) d s,
$$

where $x \wedge y=\min (x, y)$ and then

$$
\begin{aligned}
\mathbb{P}(\tau \leq t) & =\mathbb{P}\left(Y_{t}=\widetilde{Y}_{t}\right) \geq \mathbb{P}\left(T=\frac{1}{p} \log \left(\varepsilon+\mathrm{e}^{p \widetilde{T}}\right), T<t\right) \\
& \geq 1-\frac{1}{2}\left(\Phi_{y}(t, 0)+\Phi_{y}(t, \varepsilon)+\int_{0}^{t}\left|f_{y}(s, 0)-f_{y}(s, \varepsilon)\right| d s\right) .
\end{aligned}
$$

The following upper bound is easily obtained, for any $0 \leq \varepsilon \leq \bar{\varepsilon}$ and any $0 \leq y \leq \bar{y}$ :

$$
\Phi_{y}(s, \varepsilon) \leq C_{1} \exp \left(-\frac{q(1-p)}{p} s\right)
$$

with $C_{1}=\exp \left(\frac{-q}{p}\left(\frac{1-p}{p} \log (1-\bar{\varepsilon})+(\bar{y}+\bar{\varepsilon})\left(1-\frac{1}{1-\bar{\varepsilon}}\right)\right)\right)$. In order to apply the mean-value theorem, we differentiate $f_{y}$ with respect to $\varepsilon$. After some computations, one can obtain the following upper bound:

$$
\left|\frac{\partial f_{y}}{\partial \varepsilon}(s, \varepsilon)\right| \leq C_{1} C_{2} \exp \left(-\frac{q(1-p)}{p} s\right)
$$

where

$$
\begin{aligned}
C_{2}= & \frac{q^{2}((1-p)(1-\bar{\varepsilon})+p(\bar{y}+\bar{\varepsilon}))}{p^{2}(1-\bar{\varepsilon})^{2}}\left(1 \vee\left(\frac{(2-p)(1-\bar{\varepsilon})+\bar{y}+\bar{\varepsilon}}{(1-\bar{\varepsilon})^{2}}\right)\right) \\
& +\frac{q(1-\bar{\varepsilon}+2 p(\bar{y}+\bar{\varepsilon}))}{p(1-\bar{\varepsilon})^{3}}
\end{aligned}
$$


Then, we easily have

$$
\int_{0}^{t}\left|f_{y}(s, 0)-f_{y}(s, \varepsilon)\right| d s \leq C_{1} C_{2} \varepsilon \int_{0}^{+\infty} \exp \left(-\frac{q(1-p)}{p} s\right) d s \leq \frac{p C_{1} C_{2}}{q(1-p)} \bar{\varepsilon}
$$

Combining Equations (12), (13), (14) and (15), and denoting by $C(\bar{y}, \bar{\varepsilon})=C_{1}+\frac{p C_{1} C_{2}}{2 q(1-p)}$, we have

$$
\mathbb{P}(\tau>t) \leq C(\bar{y}, \bar{\varepsilon})\left(\exp \left(-\frac{q(1-p)}{p} t\right)+\bar{\varepsilon}\right)
$$

then (11) is straightforward. This upper bound does not depend on $Y_{0}$ and $\widetilde{Y}_{0}$, so this result still holds for random starting points, provided that they belong to $[0, \bar{y}]$.

Proposition 2.1 and Lemma 2.4 are the main tools to prove the following result:

Proposition 2.5. Let $t_{0}>0$. There exists an explicit positive constant $K<+\infty$ such that, for all $t \geq t_{0}$,

$$
\left\|\mu_{t}-\widetilde{\mu}_{t}\right\|_{\mathrm{TV}} \leq K e^{-v t}, \quad \text { with } v=\frac{p-q}{2+\frac{p(p-q)}{q(1-p)}} .
$$

Proof. Let $\alpha \in(0,1)$ and $u>0$. We use the coupling from Proposition 2.1 in the domain $[0, \alpha t]$ and the coupling from Lemma 2.4 in the domain $[\alpha t, t]$. We set $\bar{\varepsilon}=\mathrm{e}^{-u t}$ and $\bar{y}=\frac{q(1-p)}{p(p-q)}+1$, and have the following inequality:

$$
\mathbb{P}(\tau \leq t) \geq \mathbb{P}\left(\left|Y_{\alpha t}-\widetilde{Y}_{\alpha t}\right| \leq \bar{\varepsilon}, Y_{\alpha t} \vee \widetilde{Y}_{\alpha t} \leq \bar{y}\right) \mathbb{P}\left(\tau \leq t|| Y_{\alpha t}-\tilde{Y}_{\alpha t} \mid \leq \bar{\varepsilon}, Y_{\alpha t} \vee \widetilde{Y}_{\alpha t} \leq \bar{y}\right)
$$

On the one hand,

$$
\begin{aligned}
\mathbb{P}\left(\left|Y_{\alpha t}-\tilde{Y}_{\alpha t}\right|>\bar{\varepsilon} \text { or } Y_{\alpha t} \vee \widetilde{Y}_{\alpha t}>\bar{y}\right) & \leq \mathbb{P}\left(\left|Y_{\alpha t}-\tilde{Y}_{\alpha t}\right|>\bar{\varepsilon}\right)+\mathbb{P}\left(Y_{\alpha t}>\bar{y}\right)+\mathbb{P}\left(\tilde{Y}_{\alpha t}>\bar{y}\right) \\
& \leq\left(\frac{\mathrm{W}_{1}\left(\mu_{0}, \widetilde{\mu}_{0}\right)}{\bar{\varepsilon}}+\frac{\mathbb{E}\left[Y_{0}\right]+\mathbb{E}\left[\widetilde{Y}_{0}\right]}{\bar{y}-\frac{q(1-p)}{p(p-q)}}\right) \exp (-\alpha(p-q) t) \\
& \leq C_{3} \exp ((u-\alpha(p-q)) t),
\end{aligned}
$$

with $C_{3}=\left(\mathrm{W}_{1}\left(\mu_{0}, \widetilde{\mu}_{0}\right)+\mathbb{E}\left[Y_{0}+\widetilde{Y}_{0}\right]\right)$. On the other hand, let $C_{4}=\sup _{t \geq t_{0}} C\left(\bar{y}, \mathrm{e}^{-u t}\right)$. The constant $C_{4}$ is finite and, from Lemma 2.4,

$$
\mathbb{P}\left(\tau>t|| Y_{\alpha t}-\widetilde{Y}_{\alpha t} \mid \leq \bar{\varepsilon}, Y_{\alpha t} \vee \widetilde{Y}_{\alpha t} \leq \bar{y}\right) \leq C_{4}\left(\mathrm{e}^{-u t}+\exp \left(-\frac{q(1-p)(1-\alpha)}{p} t\right)\right) .
$$

Now, (17) reduces to

$$
\mathbb{P}(\tau>t) \leq 1-\left(1-C_{3} \exp ((u-\alpha(p-q)) t)\right)\left(1-C_{4}\left(\mathrm{e}^{-u t}+\exp \left(-\frac{q(1-p)(1-\alpha)}{p} t\right)\right)\right) .
$$

We optimize the rate of convergence by setting

$$
\alpha=\frac{1}{1+\frac{p(p-q)}{2 q(1-p)}}, \quad u=\frac{(p-q) \alpha}{2}=v \text { as defined above. }
$$

Then, (16) holds with $K=C_{3}+2 C_{4}$. 


\section{LONG TIME SYNCHRONIZATION OF LARGE POPULATIONS OF INTERACTING NOISY ROTATORS}

\subsection{The model}

Synchronization phenomena are a subject widely studied in physics and natural sciences. Synchronization can occur in several different situations, for example in the case of interacting cardiac cells, neurons, metronomes... (see [34] for numerous examples of synchronization phenomena). To construct a mathematical model in which a synchronization phenomenon occurs, one may consider a population of dynamical systems that interact with each other, and may perturb these systems with noise (to modelize the internal noise of each dynamical system, or the noise given by the interaction of each system with the surrounding environment). We will focus here on a model given by a population of $N$ interacting noisy rotators. Each rotator is defined by a phase $\varphi_{j}(t) \in \mathbb{T}=\mathbb{R} / 2 \pi \mathbb{Z}$, and the evolution of these phases is given by the following system of stochastic differential equations:

$$
\mathrm{d} \varphi_{j}(t)=-\frac{K}{N} \sum_{i=1}^{N} \sin \left(\varphi_{j}(t)-\varphi_{i}(t)\right) \mathrm{d} t+\sigma \mathrm{d} B_{j}(t),
$$

where $K \geq 0$ and $\sigma>0$ are two constant parameters, and $\left(B_{j}\right)_{j=1, \ldots, N}$ is a family of standard independent Brownian motions. The interaction is of mean field type: the rotator $\varphi_{j}$ interacts with all the other rotators, and the interaction term is constituted of the sum of the contributions given by each one of these other rotators, with the same weight $K / N$. Remark that the model is invariant by rotation: if $\left(\varphi_{j}(t)\right)_{j=1, \ldots, N}$ is a solution of (18), then it is also the case of $\left(\varphi_{j}(t)+c\right)_{j=1, \ldots, N}$ for all real constant $c$.

This model is known in the physics literature as mean field plane rotors model, and is a particular case of the celebrated noisy Kuramoto model (when the disorder follows the trivial distribution $\delta_{0}$ ). Of course, since the dynamics of each isolated system is very simple in this model (a Brownian motion on a circle), its aim is not to describe a real phenomenon (to do this one would need some complex isolated system, in higher dimension and with several parameters), but to provide a simple framework in which one can study analytically a synchronization phenomenon.

In this model we can speak of synchronization if the rotators concentrate around some phase, which we will call center of synchronization. This may happen if the interaction is strong enough with respect to the noise (since this later one incites the rotators to move independently), or in other words if $K$ is sufficiently large compared to $\sigma$. But with a simple time change one can replace $\sigma$ by 1 , and $K$ by $K / \sigma^{2}$. So the real parameter of the model is $K / \sigma^{2}$, and we will set $\sigma=1$ in the remaining for simplicity.

We will focus on the behavior of model in the limit of infinite size of population. We will first consider the evolution on time intervals $[0, T]$ independent from $N$, when $N$ goes to infinity. We will then study the behavior of the model on longer time intervals, by making a rescaling in time depending on $N$. The content of the first part is based on the works $[4,16]$, while the second part describes the result proved in [5].

\subsection{Large populations and fixed time intervals}

We consider in this section the evolution on intervals of the type $[0, T]$, with $T$ independent from $N$. In this case, since the coefficients in (18) are smooth, we can apply to our model the classical results of the well-known theory of propagation of chaos $[14,36]$. Let us consider the empirical measure $\mu_{N, t}$ associated to the model, i.e. the $\mathcal{M}_{1}(\mathbb{T})$-valued process $\mu_{N, t}=\frac{1}{N} \sum_{i=1}^{N} \delta_{\varphi_{j}(t)}$, where $\mathcal{M}_{1}(\mathbb{T})$ denotes the space of probability measures on $\mathbb{T}$. If the initial condition $\mu_{N, 0}$ converges weekly to some $p_{0} \in \mathcal{M}_{1}$, then the process $\mu_{N, t}$ converges weakly on $[0, T]$ to the deterministic trajectory on $\mathcal{M}_{1}$ solution of the following Fokker-Planck type partially differential equation:

$$
\partial_{t} p_{t}(\theta)=\frac{1}{2} \partial_{\theta}^{2} p_{t}(\theta)-\partial_{\theta}\left[p_{t}(\theta) J * p_{t}(\theta)\right]
$$

where $*$ denotes the convolution operator and $J(\theta)=-K \sin (\theta)$. In this limit $p_{t}$ is the distribution of the infinite population of rotators on $\mathbb{T}$ at time $t$. The mass is preserved by this evolution, and due to the presence of the Laplacian 
term this PDE admits a unique solution for any initial condition $p_{0} \in \mathcal{M}_{1}$, which admits a smooth and positive density for all $t>0$ (that we will also denote $p_{t}(\theta)$ for simplicity), element of $C^{\infty}((0, T) \times \mathbb{T}, \mathbb{R})$ (see for example [4] for a proof of this regularity result). Remark that the invariance by rotation of the finite size model is conserved in the limit, since one can easily check that if $p_{t}(\theta)$ is solution of (19), then it is also the case of $p_{t}(\theta-c)$ for all $c \in \mathbb{R}$.

A pleasant property of this PDE is that one can compute all its stationary solutions in a semi-explicit way: $q$ is a (probability) stationary solution for (19) if and only if $q$ can be expressed as

$$
q(\theta)=q_{\psi, r}(\theta):=\frac{e^{2 K r \cos (\theta-\psi)}}{\int_{\mathbb{T}} e^{2 K r \cos \left(\theta^{\prime}-\psi\right)} \mathrm{d} \theta^{\prime}},
$$

for some $\psi \in \mathbb{R}$ and some solution $r \geq 0$ of the fixed point problem

$$
r=\Psi(2 K r)
$$

where the fixed point function $\Psi$ is known explicitly, and satisfies some nice properties that allow us to determine the number of solutions of the fixed point problem according to the value of $K: \Psi$ is strictly concave and bounded by 1 on $(0, \infty)$, and satisfies $\Psi(0)=0$ and $\Psi^{\prime}(0)=1 / 2$ (see [4] for more details).

First remark that the equality $\Psi(0)=0$ implies that $r=0$ is always solution to the fixed point problem (21), which means that the uniform probability on the circle $q(\theta)=\frac{1}{2 \pi}$ is always a stationary solution. This solution corresponds to a total absence of synchronization in the model, since in that case the population of rotators is distributed uniformly on the $\mathbb{T}$. Moreover, the fact that $\Psi^{\prime}(0)=1 / 2$ and the strict concavity of $\Psi$ imply that if $K \leq 1, r=0$ is the only solution of (21). When $K \leq 1$ the interaction is too weak to allow the apparition of synchronized states.

On the other hand, if $K>1$, then there exists a unique positive solution $r_{K}$ to the fixed point problem, which means that in that case the set of stationary solutions of (19) is composed of $\frac{1}{2 \pi}$ and a whole family $M$ of non-trivial stationary solutions, defined as

$$
M=\left\{q_{\psi}, \psi \in \mathbb{T}\right\}, \quad \text { where } q_{\psi}(\theta)=q_{\psi, r_{K}}(\theta) .
$$

Each stationary state $q_{\psi}$ is the translation by an angle $\psi$ of the profile $q_{0}$, which corresponds to a concentration of the rotators around the phase 0 (see figure 3.2). So $q_{\psi}$ corresponds to a synchronization of the rotators around the center of synchronization $\psi$. From a geometrical point of view $M$ is a closed curve (in fact a circle since all its points are the translation of the same profile) of synchronized stationary solutions, parametrized by their centers of synchronization.

The next step in the understanding of the model is to determine the local stability of these different stationary states. To do this let us linearize the evolution around these stationary states. If we rewrite (19) as $\partial_{t} p_{t}=F\left(p_{t}\right)$, with $F(p)=$ $\frac{1}{2} p^{\prime \prime}-(p J * p)^{\prime}$, and consider a stationary state $q$ and a smooth function $u$ satisfying $\int_{\mathbb{T}} u=0$, we can expand $F(q+u)$ as follows:

$$
F(q+u)=F(q)+\frac{1}{2} u^{\prime \prime}(\theta)-[q(\theta) J * u(\theta)+u(\theta) J * q(\theta)+u(\theta) J * u(\theta)]^{\prime} .
$$

$F(q)=0$ since $q$ is stationary, and by just keeping the linear terms we get a linearized evolution $\partial_{t} u=L_{q} u_{t}$ around the stationary profile $q$, with

$$
L_{q} u(\theta):=\frac{1}{2} u^{\prime \prime}(\theta)-[q(\theta) J * u(\theta)+u(\theta) J * q(\theta)]^{\prime} .
$$

The spectral properties of the operator $L_{q}$ determines the behavior of the solutions of (19) in the neighborhood of $q$. Its spectrum can be obtained easily when $q=\frac{1}{2 \pi}$ since in that case $L_{\frac{1}{2 \pi}}$ can be decomposed on the Fourier basis: (24) boils down to

$$
L_{\frac{1}{2 \pi}} u(\theta)=\frac{1}{2} u^{\prime \prime}(\theta)-\frac{1}{2 \pi}[J * u(\theta)]^{\prime},
$$

and by writing $u(\theta)=\sum_{k=1}^{\infty} a_{k} \cos (k \theta)+\sum_{k=1}^{\infty} b_{k} \sin (k \theta)$ one simply gets (recall that $J(\theta)=-K \sin \theta$ ):

$$
L_{\frac{1}{2 \pi}} u(\theta)=-\frac{1}{2}(1-K) a_{1} \cos \theta-\frac{1}{2}(1-K) b_{1} \sin \theta-\frac{1}{2} \sum_{k=2}^{\infty} k^{2} a_{k} \cos (k \theta)-\frac{1}{2} \sum_{k=2}^{\infty} k^{2} b_{k} \sin (k \theta) .
$$




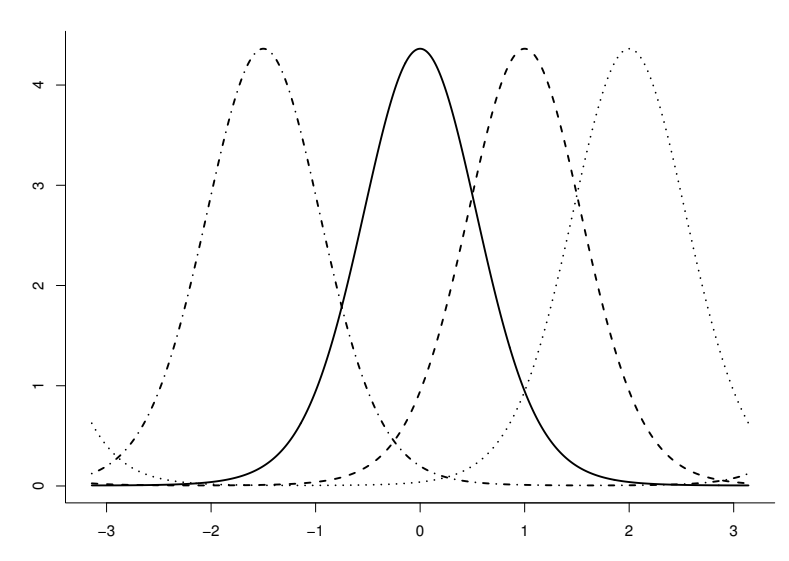

FIGURE 2. Graph of $q_{-1.5}, q_{0}, q_{1}, q_{2}$ (from the left to the right) when $K=2$. The elements of $M$ are the translations of the synchronized profile $q_{0}$.

This shows that when $K<1$ the spectrum of $L_{\frac{1}{2 \pi}}$ is composed of strictly negative eigenvalues, and thus $\frac{1}{2 \pi}$ is stable for the linearized evolution. When $K>1$ the directions given by $\cos \theta$ and $\sin \theta$ become instable.

The study of the spectrum of $L_{q_{\psi}}$ for $q_{\psi} \in M$ when $K>1$ is more involved, and requires the use of weighted Sobolev spaces. In [4] the authors show that in a well chosen weighted space the $L_{q_{\psi}}$ can also be decomposed on an orthogonal basis, and that the spectrum is made of a decreasing sequence of non positive reals $\left(\lambda_{i}\right)_{i \geq 0}$ satisfying $\lambda_{0}=0, \lambda_{i}<0$ for $i \geq 1$ and $\lambda_{i} \rightarrow_{i \rightarrow \infty}-\infty$. The eigenvalue 0 is associated to the eigenvector $q_{\psi}^{\prime}$, which generates the tangent space of $M$ at $q_{\psi}$. So the fact that the operator $L_{q_{\psi}}$ has no effect in this direction is not surprising, since in this direction the dynamics given by (19) is trivial. On the normal space (that is the subspace generated by the other eigenfunctions), the linearized dynamics is stable with a spectral gap given by the largest negative eigenvalue $\lambda_{1}$.

Knowing the existence of this spectral gap in the normal space, one can show that these spectral properties imply the local stability of the curve $M$ for the nonlinear dynamics given by (19) (see [20] for a general proof, or [16] for a proof in our particular model). In other words, if $p_{0}$ is sufficiently close to $M$, then there exists a phase $\psi$ such that $p_{t} \rightarrow t \rightarrow \infty q_{\psi}$. This shows that the synchronization is a stable phenomenon when $K>1$. But this does not mean that $q_{\psi}$ alone is stable: the trajectory starting from some perturbation $q_{\psi}+\epsilon u_{t}$ of the profile $q_{\psi}$ may converge to a profile $q_{\tilde{\psi}}$ with a phase $\tilde{\psi} \neq \psi$ (but converging to $\psi$ when $\epsilon$ goes to 0 ).

One can in fact do far better than proving the local stability of $M$, by describing completely the dynamics. Using in particular the free energy of the system [5,16], one can prove that, when $K>1$ the solutions of (19) starting from $U=\left\{p_{0} \in \mathcal{M}_{1}(\mathbb{T}): \int_{\mathbb{T}} e^{i \theta} \mathrm{d} u(\theta)=0\right\}$ converge to $\frac{1}{2 \pi}$, while if $p_{0} \notin U$ then $p_{t}$ converges to a $q_{\psi} \in M$.

\subsection{Long time behavior}

The end of the preceeding section is devoted to the long time behavior of the limit PDE (19) of the model, that is the limit in time of the model when the size of the population has already been sent to infinity. It is the long time behavior of a deterministic system, given by the limit PDE. But when $N$ is large but finite, there is still noise in the system. So, in that case, even if the model is very close to the limit PDE for some finite interval of time, its behavior can differ dramatically from the one of the limit PDE in very long times, due to the presence of this noise.

Since the noise present in the system disappears when $N$ goes to infinity, one can see in a certain sense the model with $N$ large as a noisy perturbation of the limit PDE. So when $K>1$ and the empirical measure $\mu_{N, t}$ is close to a synchronized profile $q_{\psi} \in M$ the dynamics of the finite sized model is given by a competition between the noise and 
the operator $L_{q_{\psi}}$, since this operator dominates the limit dynamics in the neighborhood of $q_{\psi} . L_{q_{\psi}}$ induces a negative feedback on the normal space, so in this direction the process has the same behavior as an infinite version of an Ornstein Uhlenbeck process $\mathrm{d} p_{t}=-\lambda p_{t}+\sqrt{\sigma} \mathrm{d} B_{t}$ with $\sigma$ small. So the empirical measure can not go far away in this direction, and stays close to $M$. On the other hand $L_{q_{\psi}}$ has no effect on the tangent space of $M$ at $q_{\psi}$, and thus the process can diffuse in this direction. This means that when $N$ is large $\mu_{N, t}$ stays close to some $q_{\psi_{t}^{N}}, \psi_{t}^{N}$ being some random phase trajectory. Of course this random phase $\psi_{t}^{N}$ converges to a constant on finite time intervals (since the process converges to a solution of the limit PDE) and thus to see a macroscopic effect of the noise in the limit one needs to rescale the time. Since the noise is of size $\sqrt{t / N}$, the appropriate renormalization is to look at times of order $N$. It is the purpose of the following Theorem, which corresponds to Theorem 1.1 in [5]. We denote $\|\cdot\|_{-1}$ the $H^{-1}$ norm.

Theorem 3.1. Suppose that $K>1$ and let $\tau_{f}>0$ and $\psi_{0} \in \mathbb{T}$. If for all $\epsilon>0$

$$
\lim _{N \rightarrow \infty} \mathbb{P}\left(\left\|\mu_{N, 0}-q_{\psi_{0}}\right\|_{-1} \leq \epsilon\right)=1,
$$

then there exists a continuous process $\left(\psi_{\tau}^{N}\right)_{\tau \geq 0}$ adapted to the filtration generated by the sequence $\left(B_{N .}^{j}\right)_{j=1, \ldots, N}$ such that for all $\epsilon>0$

$$
\lim _{N \rightarrow \infty} \mathbb{P}\left(\sup _{\tau \in\left[0, \tau_{f}\right]}\left\|\mu_{N, \tau N}-q_{\psi_{\tau}^{N}}\right\|_{-1} \leq \epsilon\right)=1,
$$

and such that $\psi_{t}^{N}$ converges weakly to $\psi_{0}+D_{K} W_{t}$, where $\left(W_{t}\right)_{t \geq 0}$ is a standard Brownian motion and $D_{K}$ is a constant that can be computed explicitly (in terms of the positive solution $r_{K}$ of the fixed point problem (21)).

The proof given in [5] of this Theorem is based on a discretization of the dynamics on an intermediate time-scale, and on a projection of the empirical measure on $M$ at each time step to follow the fluctuations of the center of synchronization induced by the noise. This procedure is inspired from the works $[3,8]$, where the authors show the diffusive behavior of the phase boundary for a one dimensional reaction-diffusion model with bistable potential and perturbed with a white noise. This discretization done, one of the main difficulties in the proof is then to show that the dynamics of this discretized phase dynamics does not contain any drift term in the limit $N \rightarrow \infty$, and this is obtained with use of the symmetry properties of the model: its invariance by rotation has already been pointed out before, but one can also remark that if $p_{t}(\theta)$ is a solution of (19), then it is also the case of $p_{t}(-\theta)$. See [5] for more details.

\section{WASSERSTEIN STABILITY OF TRAVELING WAVES FOR SCALAR NONLINEAR ADVECTION-DIFFUSION EQUATIONS}

This section addresses the long time behaviour of the scalar nonlinear advection-diffusion equation

$$
\partial_{t} u+\partial_{x}(B(u))=\frac{1}{2} \partial_{x}^{2}(A(u)), \quad t \geq 0, \quad x \in \mathbb{R},
$$

where $A$ and $B$ are $\mathrm{C}^{1}$ functions, and $A^{\prime}(u)=\sigma^{2}(u) \geq 0$. In particular, we aim at illustrating, and extending to Wasserstein distances, the classical L ${ }^{1}$ stability results of traveling waves of (29), which go back to Osher and Ralston [33] as well as Freistühler and Serre [12]. We will use probabilistic arguments, based on the interpretation by Jourdain and coauthors $[23,25,26]$ of $(29)$ as the Fokker-Planck equation of a nonlinear diffusion process. The connection between the long time behaviour of this process and the traveling waves of (29) was recently pointed out to the author of this survey by Julien Vovelle, to whom warm thanks are due.

\subsection{Traveling waves and stationary solutions to (29)}

When $\sigma^{2}$ is constant, the equation (29) is a viscous scalar conservation law. A (bounded) traveling wave for this equation is a function $\phi$ solving

$$
\frac{\sigma^{2}}{2} \phi^{\prime}=B(\phi)-s \phi-q, \quad \lim _{x \rightarrow \pm \infty} \phi(x)=w^{ \pm}
$$


where the speed of the wave $s$ and $w^{-} \neq w^{+}$satisfy the Rankine-Hugoniot condition

$$
s=\frac{B\left(w^{+}\right)-B\left(w^{-}\right)}{w^{+}-w^{-}},
$$

which implies $q=B\left(w^{ \pm}\right)-s w^{ \pm}$. In the sequel, the boundedness will be implicitly assumed and therefore $\phi$ will only be referred to as a traveling wave.

By the Cauchy-Lipschitz Theorem, for a solution to (30) to exist, it is necessary and sufficient that $q \neq B(w)-s w$ for $w$ strictly between $w^{-}$and $w^{+}$, which is usually called the Oleunnik $E$-condition [32]. Under this condition, all the traveling waves are translations of each other. If $w^{-}<w^{+}$, the Oleŭnik $E$-condition rewrites

$$
\forall w \in\left(w^{-}, w^{+}\right), \quad \frac{B(w)-B\left(w^{-}\right)}{w-w^{-}}>\frac{B\left(w^{+}\right)-B\left(w^{-}\right)}{w^{+}-w^{-}},
$$

that is to say the graph of $B$ remains strictly above the line segment joining the points $\left(w^{-}, B\left(w^{-}\right)\right)$and $\left(w^{+}, B\left(w^{+}\right)\right)$, and it implies that $\phi^{\prime}>0$ on $\mathbb{R}$, so that $\phi$ increases from $w^{-}$to $w^{+}$. If $w^{-}>w^{+}$, then the graph of $B$ must remain strictly below this line segment, and $\phi$ decreases from $w^{-}$to $w^{+}$.

If $\phi$ is a traveling wave of (29), it is immediate that $u(t, x):=\phi(x-s t)$ solves (29). If $s=0$, then $u$ is a stationary solution to (29). It has been known since the works by Il'in and Oleĭnik [21,22] that traveling waves describe the long time behavior of solutions to (29). The following $\mathrm{L}^{1}$ stability theorem is due Freistühler and Serre [12] and is based on a former result by Osher and Ralston [33].

Theorem 4.1 (Freistühler and Serre). Let $\phi$ be a traveling wave of (29) and $u_{0}$ such that $u_{0}-\phi \in \mathrm{L}^{1}(\mathbb{R})$. The solution $u(t, x)$ of (29) with initial datum $u_{0}$ satisfies

$$
\lim _{t \rightarrow+\infty}\|u(t, \cdot)-\phi(\cdot-s t+\delta)\|_{\mathrm{L}^{1}(\mathbb{R})}=0,
$$

where the phase shift $\delta$ is defined by

$$
\delta:=\frac{1}{w^{+}-w^{-}} \int_{x \in \mathbb{R}}\left(u_{0}(x)-\phi(x)\right) \mathrm{d} x .
$$

Note that the definition (34) of $\delta$ ensures that

$$
\int_{x \in \mathbb{R}}\left(u_{0}(x)-\phi(x+\delta)\right) \mathrm{d} x=0 .
$$

For a nonconstant diffusion coefficient $\sigma^{2}$, the equation (30) defining a traveling wave has to be replaced with

$$
\frac{1}{2}(A(\phi))^{\prime}=B(\phi)-s \phi-q, \quad \lim _{x \rightarrow \pm \infty} \phi(x)=w^{ \pm},
$$

where $s$ is still defined in terms of $w^{-} \neq w^{+}$by the Rankine-Hugoniot condition (31). Under the uniform ellipticity condition $\inf _{u} \sigma^{2}(u)>0$, the Oleŭnik $E$-condition remains necessary and sufficient for a traveling wave to exist, and traveling waves remain monotonic on the real line. Gasnikov [15] proved that, if $A$ and $B$ are $\mathrm{C}^{4}$ on $\left[w^{-}, w^{+}\right]$(or $\left.\left[w^{+}, w^{-}\right]\right)$, then Theorem 4.1 holds without any change in its statement.

\subsection{Probabilistic interpretation of (29)}

Traveling waves to (29) with $w^{-}=0$ and $w^{+}=1$ can be interpreted as cumulative distribution functions (CDFs) of probability measures on the real line. If we assume that the initial datum $u_{0}$ of (29) is also the CDF of a probability 
measure $m$, then $u(t, \cdot)$ remains the CDF of a probability measure $P_{t}$ at all times. Besides, taking the space derivative of (29) yields the formal evolution equation

$$
\partial_{t} P_{t}=\frac{1}{2} \partial_{x}^{2}\left(\sigma^{2}\left(H * P_{t}(x)\right) P_{t}\right)-\partial_{x}\left(b\left(H * P_{t}(x)\right) P_{t}\right)
$$

for $P_{t}$, where $b:=B^{\prime}$ and $H * P_{t}=u(t, \cdot)$ refers to the spatial convolution of $P_{t}$ with the Heaviside function. The equation (37) is the Fokker-Planck equation of the diffusion process

$$
\left\{\begin{array}{l}
\mathrm{d} X_{t}=b\left(H * P_{t}\left(X_{t}\right)\right) \mathrm{d} t+\sigma\left(H * P_{t}\left(X_{t}\right)\right) \mathrm{d} W_{t}, \\
P_{t} \text { is the law of } X_{t},
\end{array}\right.
$$

where $\left(W_{t}\right)_{t \geq 0}$ is a standard real Brownian motion, and $X_{0}$ is distributed according to $m$, independently of $\left(W_{t}\right)_{t \geq 0}$. Note that the coefficients of this stochastic differential equation depend on the law $P_{t}$ of $X_{t}$, which is the trace of the nonlinearity of the Fokker-Planck equation (37). Therefore, the process $\left(X_{t}\right)_{t \geq 0}$ is said to be nonlinear in McKean's sense.

The existence and uniqueness of the nonlinear process $\left(X_{t}\right)_{t \geq 0}$ were established in [26] under the assumptions that $b$ and $\sigma^{2}$ are continuous on $[0,1], m$ have a finite first order moment, $\sigma^{2}(u)>0$ on $(0,1)$ and:

- if $\sigma^{2}(0)=0$, then $u_{0}(x)>0$ for all $x \in \mathbb{R}$;

- if $\sigma^{2}(1)=0$, then $u_{0}(x)<1$ for all $x \in \mathbb{R}$;

where $u_{0}:=H * m$ is the $\mathrm{CDF}$ of the initial distribution $m$.

Remark 4.2. The assumption on the finiteness of the first order moment of $m$ is natural in order to obtain $\mathrm{L}^{1}$ stability results on the solution to (29). Indeed, in general, if $F_{1}$ and $F_{2}$ are the CDFs of probability measures $m_{1}$ and $m_{2}$ on $\mathbb{R}$, then $\left\|F_{1}-F_{2}\right\|_{\mathrm{L}^{1}(\mathbb{R})}$ need not be finite, but if we assume in addition that the first order moments of $m_{1}$ and $m_{2}$ are finite, then $\left\|F_{1}-F_{2}\right\|_{\mathrm{L}^{1}(\mathbb{R})}<+\infty$, and the difference between the expectations of $m_{1}$ and $m_{2}$ is given by

$$
\int_{x \in \mathbb{R}} x m_{1}(\mathrm{~d} x)-\int_{x \in \mathbb{R}} x m_{2}(\mathrm{~d} x)=\int_{x \in \mathbb{R}}\left(F_{1}(x)-F_{2}(x)\right) \mathrm{d} x .
$$

We first provide a probabilistic interpretation of the speed $s$ of a traveling wave as the average velocity of the nonlinear process $\left(X_{t}\right)_{t \geq 0}$. Indeed, the expectation of $X_{t}$ satisfies

$$
\mathbb{E}\left[X_{t}\right]=\mathbb{E}\left[X_{0}\right]+\int_{s=0}^{t} \mathbb{E}\left[b\left(H * P_{s}\left(X_{s}\right)\right)\right] \mathrm{d} s .
$$

Besides, it was proved in [26] that, $\mathrm{d} s$-almost everywhere, the measure $P_{s}$ does not weight points, which implies that $H * P_{s}\left(X_{s}\right)$ is uniformly distributed on $[0,1]$. We therefore rewrite

$$
\mathbb{E}\left[X_{t}\right]=\mathbb{E}\left[X_{0}\right]+\int_{s=0}^{t} \int_{u=0}^{1} b(u) \mathrm{d} u \mathrm{~d} s=\mathbb{E}\left[X_{0}\right]+s t,
$$

where $s$ is given by the Rankine-Hugoniot condition $s=B(1)-B(0)$.

We now describe the long time behaviour of the nonlinear process in terms of traveling waves. We first discuss conditions ensuring that traveling waves are well defined thanks to the following lemma, which was obtained in [26, Proposition 4.1 and Corollary 4.4] by solving (36) explicitly.

Lemma 4.3. Assume that $b$ and $\sigma^{2}$ are continuous on $[0,1]$, that $A$ is increasing on $[0,1]$, and that the Oleunik E-condition

$$
\forall u \in(0,1), \quad B(u)>B(0)+s u
$$

is satisfied, where $s$ is defined by the Rankine-Hugoniot condition $s=B(1)-B(0)$. 
Then there exists a traveling wave $\phi$ increasing from 0 to 1 , and all such traveling waves are translations of each other. Besides, the probability measure with $C D F \phi$ has a finite first order moment if and only if

$$
\int_{u=0}^{1 / 2} \frac{u \sigma^{2}(u)}{B(u)-B(0)-s u} \mathrm{~d} u+\int_{u=1 / 2}^{1} \frac{(1-u) \sigma^{2}(u)}{B(u)-B(0)-s u} \mathrm{~d} u<+\infty .
$$

Let us mention that, if the Oleunik $E$-condition is relaxed by allowing that $B(u)=B(0)+s u$ for some $u \in(0,1)$ such that $\sigma^{2}(u)=0$, then one can exhibit traveling waves that are not translations of each other, see [26, Remark 4.2].

Let us now fix a probability measure $m$ with finite first order moment, and let $\phi$ be given by Lemma 4.3. If (43) holds, then by Remark 4.2, $u_{0}-\phi \in \mathrm{L}^{1}(\mathbb{R})$; besides, choosing the phase shift $\delta$ in order to satisfy (35) amounts to selecting $u_{\infty}=\phi(\cdot+\delta)$ having the same expectation as $u_{0}$. By (41), we already know that the expectation of $X_{t}-s t$ is constant and equal to the expectation of $u_{\infty}$. Theorem 4.1 contains the much stronger statement that the long time behaviour of this process is described by the stationary wave $u_{\infty}$, in the sense that the CDF of $X_{t}-s t$ converges to $u_{\infty}$ in $\mathrm{L}^{1}(\mathbb{R})$. In the next subsection, we extend this result to Wasserstein distances.

\subsection{Contraction and convergence to equilibrium in Wasserstein distance}

We recall that the Wasserstein distance of order $p \in[1,+\infty)$ between two probability measures on the real line with respective $\mathrm{CDFs} F_{1}$ and $F_{2}$ is given by

$$
\mathrm{W}_{p}\left(F_{1}, F_{2}\right):=\left(\int_{w=0}^{1}\left|F_{1}^{-1}(w)-F_{2}^{-1}(w)\right|^{p} \mathrm{~d} w\right)^{1 / p},
$$

where the pseudo-inverse $F^{-1}$ of a $\operatorname{CDF} F$ is defined by $F^{-1}(w):=\inf \{x \in \mathbb{R}: F(x) \geq w\}$ for all $w \in(0,1)$. Note that, in particular,

$$
\mathrm{W}_{1}\left(F_{1}, F_{2}\right)=\left\|F_{1}-F_{2}\right\|_{\mathrm{L}^{1}(\mathbb{R})} .
$$

Our first result is the following Wasserstein contraction property of (29). Given two CDFs $u_{0}, v_{0}$ on the real line, we now denote by $u_{t}:=u(t, \cdot)$ and $v_{t}:=v(t, \cdot)$ the corresponding solutions to (29).

Proposition 4.4. Assume that $b$ and $\sigma^{2}$ are continuous on $[0,1]$, that $A$ is increasing on $[0,1]$ and that $m$ has a finite first order moment. For all $p \in[1,+\infty)$,

- if $\mathrm{W}_{p}\left(u_{0}, v_{0}\right)=+\infty$, then $\mathrm{W}_{p}\left(u_{t}, v_{t}\right)=+\infty$ for all $t \geq 0$;

- if $\mathrm{W}_{p}\left(u_{0}, v_{0}\right)<+\infty$, then $t \mapsto \mathrm{W}_{p}\left(u_{t}, v_{t}\right)$ is nonincreasing on $[0,+\infty)$.

The proof of Proposition 4.4 is detailed in [26, Proposition 3.1]. It is entirely probabilistic, and relies on a coupling argument for the order statistics of a system of mean-field interacting particles approximating $u_{t}$ and $v_{t}$. We note that, by (45), the case $p=1$ of Proposition 4.4 is nothing but the classical $\mathrm{L}^{1}$ stability estimate

$$
\forall t \geq 0, \quad\left\|u_{t}-v_{t}\right\|_{\mathrm{L}^{1}(\mathbb{R})} \leq\left\|u_{0}-v_{0}\right\|_{\mathrm{L}^{1}(\mathbb{R})}
$$

for (29). Similar Wasserstein estimates were obtained for scalar conservation laws, that is to say $\sigma^{2}=0$ in our setting, by Bolley, Brenier and Loeper in [6].

Assuming classical regularity for $u$ and $v$, one can actually go deeper into the description of the evolution of $\mathrm{W}_{p}\left(u_{t}, v_{t}\right)$. Indeed, observing that the pseudo-inverse $u_{t}^{-1}(w)$ of the $\mathrm{CDF} u_{t}$ satisfies the equation

$$
\partial_{t} u_{t}^{-1}=b(w)-\partial_{w}\left(\frac{\sigma^{2}(w)}{2 \partial_{w} u_{t}^{-1}}\right)
$$

one can derive the explicit formula for the time derivative of $\mathrm{W}_{p}\left(u_{t}, v_{t}\right)$ :

$$
\frac{\mathrm{d}}{\mathrm{d} t} \mathrm{~W}_{p}\left(u_{t}, v_{t}\right)^{p}=-\frac{p(p-1)}{2} \int_{w=0}^{1} \sigma^{2}(w)\left|u_{t}^{-1}-v_{t}^{-1}\right|^{p-2} \frac{\left(\partial_{w} u_{t}^{-1}-\partial_{w} v_{t}^{-1}\right)^{2}}{\partial_{w} u_{t}^{-1} \partial_{w} v_{t}^{-1}} \mathrm{~d} w .
$$


This leads to the following convergence theorem, which is the main result of [26] and readily extends Theorem 4.1 to Wasserstein distances.

Theorem 4.5. Assume that:

- $b$ is $\mathrm{C}^{1+\beta}$ on $[0,1], \sigma^{2}$ is positive and $\mathrm{C}^{2+\beta}$ on $[0,1]$, and the equilibrium conditions (42) and (43) hold;

- the probability measure $m$ has a finite first order moment;

- the Wasserstein distance of order 2 between $u_{0}:=H * m$ and any traveling wave $\phi$ increasing from 0 to 1 is finite.

Let us denote by $u_{\infty}$ the traveling wave with the same expectation as $u_{0}$. Then, for all $p \geq 2$ such that $\mathrm{W}_{p}\left(u_{0}, u_{\infty}\right)<$ $+\infty$, we have

$$
\forall 1 \leq q<p, \quad \lim _{t \rightarrow+\infty} \mathrm{W}_{q}\left(u(t, \cdot), u_{\infty}(\cdot-s t)\right)=0 .
$$

\subsection{Conclusion}

We have interpreted the scalar nonlinear advection-diffusion equation (29), with a CDF as an initial datum, as the Fokker-Planck equation of a nonlinear diffusion process $\left(X_{t}\right)_{t \geq 0}$. The expectation of this process evolves linearly in time, at a velocity given by the speed of traveling waves of (29) increasing from 0 to 1 . Under the Olěnik $E$-condition (42), Theorem 4.1 shows that the fluctuation of $X_{t}$ around st converges, in $\mathrm{L}^{1}(\mathbb{R})$, to an equilibrium distribution described by the traveling wave having the same expectation as $X_{t}-s t$. Theorem 4.5 extends this result to Wasserstein distance.

The probabilistic interpretation of (29) can also lead to further developments on Theorems 4.1 and 4.5. For example, in the case of a constant diffusion coefficient $\sigma^{2}$, an exponential rate of decay to equilibrium for $X_{t}-s t$ was obtained in [25],

for initial distributions close to the equilibrium distribution. The decay was expressed in $\chi_{2}$ distance, and using the transport chi-square inequality of [24], it can be translated in quadratic Wasserstein distance. We refer to [26, Subsection 4.3] for details in this direction.

\section{REFERENCES}

[1] J.-B. Bardet, A. Christen, A. Guillin, F. Malrieu, and P.-A. Zitt. Total variation estimates for the TCP process. Electron. J. Probab, 18(10):1-21, 2013.

[2] P. Bertail, S. Clémençon, and J. Tressou. A storage model with random release rate for modeling exposure to food contaminants. Math. Biosci. Eng., 5(1):35-60, 2008.

[3] L. Bertini, S. Brassesco, and P. Buttà. Soft and hard wall in a stochastic reaction diffusion equation. Arch. Ration. Mech. Anal., 190:307-345, 2008.

[4] L. Bertini, G. Giacomin, and K. Pakdaman. Dynamical aspects of mean field plane rotators and the Kuramoto model. Journal of Statistical Physics, 138:270-290, 2010.

[5] L. Bertini, G. Giacomin, and C. Poquet. Synchronisation and random long time dynamics for mean-field plane rotators. Probab. Theory Relat. Fields, 2013.

[6] F. Bolley, Y. Brenier, and G. Loeper. Contractive metrics for scalar conservation laws. J. Hyperbolic Differ. Equ., 2(1):91-107, 2005.

[7] F. Bouguet. Quantitative speeds of convergence for exposure to food contaminants. Preprint available on arXiv, to appear in ESAIM P\& S, 2013.

[8] S. Brassesco, A. De Masi, and E. Presutti. Brownian fluctuations of the interface in the $d=1$ Ginzburg-Landau equation with noise. Annal. Inst. H. Poincaré, 31:81-118, 1995.

[9] M. H. A. Davis. Markov models and optimization, volume 49 of Monographs on Statistics and Applied Probability. Chapman \& Hall, London, 1993.

[10] D. Down, S. P. Meyn, and R. L. Tweedie. Exponential and uniform ergodicity of Markov processes. Ann. Probab., 23(4):1671-1691, 1995.

[11] J. Fontbona and F. Panloup. Rate of convergence to equilibrium of fractional driven stochastic differential equations with some multiplicative noise. Preprint available at http://hal.archives-ouvertes.fr/hal-00989414, pages 1-34, 2014.

[12] H. Freistühler and D. Serre. $L^{1}$ stability of shock waves in scalar viscous conservation laws. Comm. Pure Appl. Math., 51(3):291-301, 1998.

[13] S. Gadat, F. Panloup, and S. Saadane. Regret bounds for narendra-shapiro bandit algorithms. Preprint available on arXiv n ${ }^{\circ} 1502.04874,2015$.

[14] J. Gärtner. On McKean-Vlasov limit for interacting diffusions. Math. Nachr., 137:197-248, 1988.

[15] A. V. Gasnikov. Time-asymptotic behavior of the solution of the initial Cauchy problem for a conservation law with nonlinear divergent viscosity. Izv. Ross. Akad. Nauk Ser. Mat., 73(6):39-76, 2009.

[16] G. Giacomin, K. Pakdaman, and X. Pellegrin. Global attractor and asymptotic dynamics in the Kuramoto model for coupled noisy phase oscillators. Nonlinearity, 25:1247-1273, 2012.

[17] M. Hairer. Ergodicity of stochastic differential equations driven by fractional Brownian motion. Ann. Probab., 33(2):703-758, 2005.

[18] M. Hairer and A. Ohashi. Ergodic theory for SDEs with extrinsic memory. Ann. Probab., 35(5):1950-1977, 2007.

[19] M. Hairer and N. S. Pillai. Regularity of laws and ergodicity of hypoelliptic SDEs driven by rough paths. Ann. Probab., 41(4):2544-2598, 2013. 
[20] D. Henry. Geometric theory of semilinear parabolic equations, volume 840 of Lecture Notes in Mathematics. Springer-Verlag, 1981.

[21] A. M. Il'in and O. A. Olen̆nik. Behavior of solutions of the Cauchy problem for certain quasilinear equations for unbounded increase of the time. Dokl. Akad. Nauk SSSR, 120:25-28, 1958.

[22] A. M. Il'in and O. A. Oler̆nik. Asymptotic behavior of solutions of the Cauchy problem for some quasi-linear equations for large values of the time. Mat. Sb. (N.S.), 51 (93):191-216, 1960.

[23] B. Jourdain. Diffusion processes associated with nonlinear evolution equations for signed measures. Methodol. Comput. Appl. Probab., 2(1):69-91, 2000.

[24] B. Jourdain. Equivalence of the Poincaré inequality with a transport-chi-square inequality in dimension one. Electron. Commun. Probab., 17:no. 43, 12, 2012.

[25] B. Jourdain and F. Malrieu. Propagation of chaos and Poincaré inequalities for a system of particles interacting through their CDF. Ann. Appl. Probab., 18(5):1706-1736, 2008.

[26] B. Jourdain and J. Reygner. Propagation of chaos for rank-based interacting diffusions and long time behaviour of a scalar quasilinear parabolic equation. Stochastic Partial Differential Equations: Analysis and Computations, 1(3):455-506, 2013.

[27] D. Lamberton and G. Pagès. How fast is the bandit? Stoch. Anal. Appl., 26(3):603-623, 2008.

[28] D. Lamberton and G. Pagès. A penalized bandit algorithm. Electron. J. Probab., 13:no. 13, 341-373, 2008.

[29] D. Lamberton, G. Pagès, and P. Tarrès. When can the two-armed bandit algorithm be trusted? Ann. Appl. Probab., 14(3):1424-1454, 2004.

[30] T. Lindvall. Lectures on the coupling method. Dover Publications Inc., Mineola, NY, 2002. Corrected reprint of the 1992 original.

[31] D. Nualart and A. Răşcanu. Differential equations driven by fractional Brownian motion. Collect. Math., 53(1):55-81, 2002.

[32] O. A. Oleŭnik. Uniqueness and stability of the generalized solution of the Cauchy problem for a quasi-linear equation. Uspehi Mat. Nauk, 14(2 (86)):165-170, 1959.

[33] S. Osher and J. Ralston. $L^{1}$ stability of travelling waves with applications to convective porous media flow. Comm. Pure Appl. Math., 35(6):737$749,1982$.

[34] A.S. Pikovsky and J. Kurths. Coherence resonance in a noise driven excitable system. Phys. Rev. Lett., 78:775-778, 1997.

[35] S. Rădulescu and M. Rădulescu. An application of Hadamard-Lévy’s theorem to a scalar initial value problem. Proc. Amer. Math. Soc., 106(1):139143, 1989.

[36] A.-S. Sznitman. Topics in propagation of chaos. In École d'été de probabilités de Saint-Flour XIX-1989, volume 1464 of Lecture Notes in Math. Springer, 1991. 